\title{
Virological investigations of acute encephalopathy in India
}

Rashmi Kumar, Asha Mathur, Arvind Kumar, Gagan Deep Sethi, Sandhya Sharma, U C Chaturvedi

\begin{abstract}
A total of 740 consecutive children aged between 6 months and 12 years who presented with acute encephalopathic illnesses during a three year period were assessed both clinically and by laboratory investigations. Cerebrospinal fluid was examined for the presence of cells or other abnormal substances, and any organisms were cultured. Blood examination included white cell count and estimations of haemoglobin, urea, glucose, and electrolyte concentrations and serum alanine aminotransferase and aspartate aminotransferase. A firm diagnosis was established in $\mathbf{2 7 8}$ patients $(38 \%)$. Pyogenic meningitis $(n=134)$, measles encephalopathy $(n=38)$, and electrolyte imbalance $(n=23)$ were important causes in this group, cerebral malaria $(n=4)$ was uncommon and there were no cases of Reye's syndrome. The diagnoses of the remaining 462 were combined under the heading 'acute unexplained encephalopathy'. Altogether 394 of the 462 patients underwent virological investigations for arboviruses and $92(23 \%)$ had one or more indicators of Japanese encephalitis. No other arboviruses could be isolated. Throat swabs from 187 patients with acute unexplained encephalopathy were studied on monkey kidney tissue cell lines of which 14 were positive ( $8 \%$ ). These were identified as adenovirus, parainfluenza, influenza, poliomyelitis, Coxsackie, and echovirus; in two cases the virus was untypable.

Japanese encephalitis is an important cause of acute childhood encephalopathy in this region. Clinical features of the illness may be mimicked by several disorders which require specific treatment. Thirty four of the 92 died (37\%).
\end{abstract}

Acute encephalopathy is an important cause of admission to hospital and death as well as being a public health problem in India. ${ }^{1}$ Several unrelated disorders (such as bacterial and viral infections of the central nervous system, Reye's syndrome, cerebral malaria, and electrolyte imbalance) may present as acute encephalopathy in children. There were, however, no comprehensive, systematic studies of the aetiology of childhood encephalopathies in India, and in 1979 the Indian Council of Medical Research put forward recommendations for the conduct of research on these encephalopathies. ${ }^{1}$

The present study was designed to investigate the aetiology of acute childhood encephalopathy by examining all those who presented to a large city hospital in northern India where it is an important problem.

\section{Patients and methods}

Consecutive children between 6 months and 12 years of age admitted to the paediatric wards of the King George's Medical College, Lucknow, were included in the study if the main complaint was an acute constant depression of consciousness lasting for more than 12 hours, and the total duration of illness at the time of admission was one week or less.

The King George's Medical College is a large teaching hospital that caters mainly for the lower socioeconomic group and seriously ill patients from Lucknow city and district as well as the surrounding districts. About two thirds of the patients admitted come from rural areas.

Histories were taken on admission and the information was entered on a proforma, together with the results of the physical examination. A careful record of the patients' progress in hospital was maintained. An attempt was made to determine the cause of the illness from the results of the following investigations: the cerebrospinal fluid was examined for the presence of cells, protein, glucose, and organisms; full blood counts were done including examination of a peripheral smear for the presence of Plasmodium falciparum and serum concentrations of urea, glucose, and electrolytes were estimated together with the activities of alanine aminotransferase and aspartate aminotransferase. Enzyme activities were estimated when there was no sign of pleocytosis in the cerebrospinal fluid, and when no other cause could be found for the encephalopathy. Blood was cultured when indicated.

Other investigations such as computed tomography were done when indicated and possible. Patients whose diagnosis was not evident after these investigations had been carried out were termed 'acute unexplained encephalopathy' and virological investigation was carried out

The following samples were collected for virological investigations: cerebrospinal fluid; brain tissue whenever possible by necropsy brain biopsy; serum samples during the acute and convalescent phases, and throat swabs in minimum essential medium.

All samples were kept at $2-8^{\circ} \mathrm{C}$ and transported within 24 hours in ice to the virology laboratory of the King George's Medical College, which is one of the national reference laboratories for arboviruses. Viral isolation was attempted from cerebrospinal fluid and brain 
tissue by intracerebral inoculation into 1-2 day old mice. Identification of the virus was done by the quick complement fixation test using the brain suspension of sick mice as a crude antigen against hyperimmune sera of West Nile virus, ${ }^{2}$ Dengue virus serotypes 1, 2, 3, and 4, Japanese encephalitis, and Chikangunya viruses. These samples were obtained from the National Institute of Virology, Pune.

The haemagglutination inhibition test for Japanese encephalitis was done on paired serum samples. ${ }^{3} 4 \mathrm{~A}$ four fold or higher rise in the antibody titre of Japanese encephalitis virus was considered to be highly suggestive of the infection. Serum with positive haemagglutination inhibition (that is, a fourfold or higher rise in haemagglutination inhibition antibodies in paired samples) were retested after treatment with 2-mercaptoethanol to look for specific antiJapanese encephalitis virus $\operatorname{IgM}$ antibodies. Patients with both a positive haemagglutination inhibition test and detectable anti-Japanese encephalitis virus IgM were considered to have positive serology. Details of virological methods have been described previously. ${ }^{5}$

Throat swabs were inoculated into primary monolayer cultures of renal epithelial cells from Rhesus monkeys and examined for their cytopathogenic effect according to standard virological methods. Identification of viruses was done by the haemadsorption inhibition and neutralisation tests in tissue culture using standard hyperimmune serum samples obtained from Wellcome Reagents, London, and the Haffkine Institute, Bombay. ${ }^{7}$

\section{Results}

During the three year period August 1985 to August 1988 a total of 740 children was enrolled in the study. Another 42 children satisfied the study criteria but could not be investigated because they died too soon after reaching the hospital or left against medical advice. None of these children had clinical features that suggested the cause of their encephalopathy (for example, poisoning, head injury, or hypertension). In 278 of the 740 patients investigated (38\%) a firm diagnosis was reached after the cli-

Table 1 Diagnoses in 370 patients

\begin{tabular}{lc}
\hline Confirmed diagnoses & No of patients \\
\hline Bacterial meningitis & 134 \\
Tuberculous meningitis & 21 \\
Measles encephalopathy & 38 \\
Electrolyte imbalance & 23 \\
Prolonged coma after seizures & 16 \\
Hepatic encephalopathy & 8 \\
Shigellosis & 8 \\
Poisoning & 7 \\
Mumps encephalopathy & 5 \\
Miscellaneous & 5 \\
Cerebral malaria & 4 \\
Enteric encephalopathy & 4 \\
Hypertensive encephalopathy & 3 \\
Head injury & 2 \\
Reye's syndrome* & 0 \\
\hline Total confirmed diagnoses & 278 \\
Japanese encephalitis & 92 \\
\hline Total & 370 \\
\hline
\end{tabular}

${ }^{*}$ Electrolyte concentrations were estimated in 412 patients and serum enzyme activities in 414 .
Table 2 Results of virological investigations

\begin{tabular}{lr}
\hline No of patients with possible viral encephalitis & 462 \\
No of patients examined for arboviruses & 394 \\
No of patients with indicators of Japanese & 92 \\
encephalitis infection: & 5 \\
Virus isolated from brain alone & 22 \\
Virus isolated from cerebrospinal fluid alone & 59 \\
Positive serology alone & 6 \\
Positive serology and isolation of virus & \\
No of patients examined for other viruses (by throat & \\
swab inoculation into monkey kidney tissue cells) & 187 \\
No of patients positive for other viruses: & 14 \\
Adenovirus & 5 \\
Parainfluenza & 3 \\
Influenza & 1 \\
Polio & 1 \\
Coxsackie & 1 \\
Echo & 1 \\
Untypable & 2 \\
\hline
\end{tabular}

nicopathological investigations outlined above, the various diagnoses are shown in table 1 . The diagnosis of the remaining 462 patients were grouped under the heading 'acute unexplained encephalopathy' and 394 of these underwent virological investigations for arboviruses. Viral isolation was attempted from cerebrospinal fluid in 315 patients, from brain tissue in 25 patients, and from paired serum samples (which could be only obtained from 174 patients). Throat swabs were obtained from 187 patients. Results of the virological investigations are shown in table 2 .

\section{Discussion}

One problem of the study of encephalopathies is the terminology used to describe the illness. A number of such clearly characterised diseases as pyogenic meningitis, tuberculous meningitis, and Reye's syndrome may produce similar clinical pictures of acute depression of consciousness with or without other neurological findings. For this reason we have used the general term 'acute encephalopathy' as it is less restrictive as a preliminary diagnosis of a syndrome that has many possible causes. When known causes have been excluded, the term 'acute unexplained encephalopathy' has been used.

There are few studies of this kind from the Indian subcontinent. Mehrotra et al described virological, bacteriological, haematological, and epidemiological investigations in 61 patients with 'pyrexia and disturbance of conscious state often leading to coma' ${ }^{8}$ They found local or systemic bacterial infection in 30 patients and isolated viruses from seven. They concluded that the syndrome had a varied aetiology, an important cause being acute toxic encephalopathy secondary to bacterial infection. Benakappa et al studied 64 children with a 'clinical picture of encephalitis' by serum biochemical investigations including estimation of enzyme activities and virological investigations. ${ }^{9}$ John et al studied 255 patients with acute encephalopathic illnesses and attempted to isolate viruses from throat and rectal swabs, cerebrospinal fluid, blood, urine, and tissues. ${ }^{10}$ The present study is the first systematic long term, comprehensive study of the aetiology of acute childhood encephalopathy in India and was designed to conform to the guidelines of the Indian Council of Medical Research task force recommenda- 
tions for research on encephalopathies in children. ${ }^{1}$

In this study we enrolled consecutive cases with acute encephalopathic illness, and in this sense our cases were unselected. The 42 patients who could not be studied were mostly patients with unexplained encephalopathies, as none had an obvious cause for the illness. Infants below the age of 6 months were excluded from study because young babies often present with alteration of consciousness with non-neurological disorders. Only patients with continuing alteration of consciousness for more than 12 hours were entered into the study. This excluded most cases of simple convulsions.

The diagnosis of bacterial meningitis was firmly established in 134 patients, and was made because of cerebrospinal fluid turbidity and cell count, glucose concentration, or the presence of organisms. Clinically bacterial meninigitis may be indistinguishable from encephalopathies such as cerebral malaria or viral encephalitis. Even examination of the cerebrospinal fluid may not differentiate it from these conditions, especially if antibiotics have been given. Thus a few of our patients who were later proved to have viral encephalitis had received treatment for bacterial meningitis.

Tuberculous meningitis usually has a gradual onset and patients are generally not admitted to hospital within a week of the onset of the illness. Occassionally patients have short histories and such cases may be difficult to differentiate from viral encephalitis unless more detailed studies are undertaken. Our cases of tuberculous meningitis were diagnosed from other indicators of tuberculous infection with or without computed tomographic evidence of basal exudates.

After excluding meningitis, a small but important group were those patients with measles associated encephalopathy, which may be caused either by measles virus invasion of the central nervous system or by an allergic demyelinating reaction. ${ }^{11}$ Of the 38 patients with this illness 28 presented within 10 days of the onset of the measles rash.

Cerebral malaria was found in only four patients in the present series, and was diagnosed by the presence of $P$ falciparum in the peripheral blood smear. Lucknow is not an endemic area for malaria, having an annual parasite index of less than 2 (State Malaria Department, Uttar Pradesh), which explains the low occurrence of cerebral malaria in this region.

Twenty three children had electrolyte imbalances: hyponatraemia (serum sodium less than $125 \mathrm{mmol} / \mathrm{l}, \mathrm{n}=12$ ) and hypernatraemia (serum sodium greater than $150 \mathrm{mmol} / \mathrm{l}, \mathrm{n}=11$ ). These were usually associated with diarrhoea or vomiting, or both, and were probably the cause of the encephalopathy. Another two patients had hyponatraemia but were later found to have Japanese encephalitis as well.

An important finding was that not a single case of Reye's syndrome was diagnosed during the three year study period, although 414 patients were 'screened'. Serum enzyme activities serve as a useful screening test for this disorder. Although increases in serum transami- nase activities do not by themselves establish the diagnosis, they are usually raised in almost all cases. Corey et al found increased serum aspartate transaminase activities in all their 397 patients with Reye's syndrome whereas increased serum ammonia concentration was not an invariable finding. ${ }^{12}$ It is possible that several tests may be more useful than one single test, and a few cases may have been missed, but it does seem that this illness is uncommon in Lucknow. Reye's syndrome was diagnosed in 15 of 19 children with a 'clinical picture of encephalitis' studied by estimation of serum enzyme activities in Bangalore. 9 In Delhi, 12 of 80 patients $(15 \%)$ with 'encephalitis or encephalopathy syndrome' were diagnosed as having Reye's syndrome and this was confirmed by histopathological examination ${ }^{13}$; cases have also been reported in Nagpur, ${ }^{14}$ Bombay, ${ }^{15}$ and Chandigarh. ${ }^{16}$ Our findings suggest that the aetiology of this illness may vary in different parts of the country.

Heat stroke was considered to be the cause of 'acute encephalopathy syndrome' in another hinterland (Nagpur) in the hot summer months. Sriramachari and Patoria carried out neuropathological examinations of brains removed at necropsy from 15 such children and found changes that were consistent with 'effects of pyrexial damage rather than any inflammatory process'. ${ }^{17}$ Although histopathological studies were not done in the present investigation, our patients did not have temperatures high enough to suggest this diagnosis. In addition, most of the cases presented in the late monsoon season rather than the summer.

Japanese encephalitis was first recognised in India in the mid 1950s. The first serious epidemic occurred in West Bengal in 1973, and since then several epidemics have occurred in the eastern and southern parts of the country. ${ }^{18}$ In 1978 there was a large epidemic in Uttar Pradesh, indicating inland spread of the disease. ${ }^{5}$ A report of the first 86 patients in the present study showed that Japanese encephalitis is an important cause of childhood encephalopathy in the Lucknow region, with cases occurring throughout the year. ${ }^{19}$ Specimens from our patients with unexplained encephalopathy were therefore investigated for evidence of Japanese encephalitis and related arboviruses. A total of 92 cases of Japanese encephalitis out of 394 studied were identified ( $23 \%$ ); no other arboviruses could be isolated. Japanese encephalitis is thus a serious cause of acute childhood encephalopathy in this region. Cases were seen all the year round with a peak incidence in the months after the monsoon-that is, October/November when the mosquito population is at the peak. Nearly half the cases came from the towns, and 34 of the 92 died (37\%).

Other viruses such as adenovirus, paramyxovirus, and enteroviruses were isolated from throat swabs of 14 of 187 patients examined $(8 \%)$, and may have been responsible for their illnesses. Benakappa $e t$ al found five isolates of Coxsackie virus in rectal swabs and one in cerebrospinal fluid in 64 of the cases of acute encephalopathy examined. ${ }^{9}$ John et al isolated enteroviruses from the cerebrospinal fluid of 
eight children, from brain tissue in three children, and from other sites (for example, throat swabs and rectal swabs) in nine of 255 children with encephalopathy. ${ }^{10}$ Hardas et al found enteroviruses in 14 of 90 cases of encephalitis. ${ }^{20}$ Enteroviruses do therefore occasionally seem to be responsible for viral encephalitis in this country.

The cause of encephalopathy remained undetermined in about $40 \%$ of the children despite the investigations outlined. Japanese encephalitis could not be ruled out in some of these because the rate of viral isolation from cerebrospinal fluid is not high even under the best conditions. Paired serum samples could not be obtained from many patients who either died in the acute stage of the illness or recovered rapidly and were discharged from hospital before a convalescent sample was taken. Investigation for viral infections other than Japanese encephalitis was incomplete in this study, because serological tests for antibodies to other viruses were not carried out. This would have increased our ascertainment of infection by other viruses in the same way as it did for Japanese encephalitis. Investigations were therefore restricted to arbovirus, enterovirus, and viruses affecting the respiratory tract. Other disorders such as arterial or venous occlusions, intracranial haemorrhage, metabolic disorders such as systemic carnitine deficiency, defects in amino acid synthesis or organic acidaemias, and lead intoxication may also present as encephalopathy, and may have caused the illness in some of our patients.

This study was supported by the Indian Council of Medica Research, scheme No 5/8/7/3/85-ECDI.
1 Indian Council of Medical Research Task Force Report. Recommendations for research on encephalopathy in children in India. Indian $\mathcal{f}$ Pediatr 1981; 48:255.

2 Pavri KM, Shaikh BH. A rapid method of specific identification of Japanese encephalitis-West Nile subgroup of arboviruses. Current Sci 1966;35:455-6.

3 Clarke DH, Casals J. Techniques of haemagglutination and haemagolutination inhibition with arthropod borne viruses. Am 7 Trop Med Hyg 1959;7:561-73.

4 Sever JL. Application of a microtechnique to viral serological investigations. F Immunol 1962;88:320-9.

5 Mathur A, Chaturvedi UC, Tandon HO, et al. Japanese encephalitis epidemic in Uttar Pradesh, India during 1978. encephalitis epidemic in Uttar Pradest

6 Mathur A, Singh UK, Tandon HO, Chaturvedi UC. Pattern of some viruses in acute respiratory illnesses during 1972

7 Lim KA, Melnick MB. Typing of viruses by combinations of antiserum pools. Application to typing of enteroviruses. f Immunol 1960;84:309-17.

8 Mehrotra RML, Kapoor AK, Mathur A, Khan AM, Chaturvedi UC. Acute encephalopathy: a clinicopathological study. Indian $\mathcal{f}$ Med Res 1971;59:705-14.

9 Benakappa DG, Prasad SR, Sastry NSC, George S. Acute encephalopathy syndrome in Bangalore. Indian 7 Pediatr 1983;50:121-7.

10 John TJ, Feldman RA, Patoria NK, Christopher S, George $S$. Enteroviruses and acute encephalopathy syndrome. Indian $\mathcal{F}$ Pediatr 1984;51:627-31.

11 Phillips, CF. Measles. In: Behrman RE, Vaughan VC, eds. Nelson textbook of pediatrics. 12th Ed. Philadelphia: W B Nelson textbook of pediatri

12 Corey L, Rubin RJ, Bregman D, Gregg MD. Diagnostic criteria for influenza B-associated Reye's syndrome: clinical vs pathological criteria. Pediatrics 1977;60:702-13.

13 Kalra V, Sachdev HPS, Menon PSN. Reye's syndrome in Delhi. Indian f Pediatr 1981;48:585-8.

14 John TJ, Anand Date, Patoria NK. Acute encephalopathy in children in Nagpur: similarity to Reye's syndrome. Indian 7 Pediatr 1983;50:129-32.

15 Merchant RH, Bajaj RT, Shah KN, Desai MP, Gharpure SV. Reye's syndrome in Bombay. Indian $\mathcal{F}$ Pediatr 1981;48: 581-3.

16 Chandrasekaran R, Vijay Kumar C, Baneriee K, Walai BNS, Bhagwat AS. Reye's syndrome: clinical course and follow up of survivors. Indian $\mathcal{F}$ Pediatr 1973;10:337-41.

17 Sriramachari S, Patoria NK. Pathology of acute encephalopathy syndrome in children in summer. Indian $f$ Med Res pathy syndrome in

18 Medappa N. Japanese encephalitis in India. Indian Council of Medical Research Bulletin 1980;10:29-38.

19 Kumar R, Mathur A, Kumar A, Sharma S, Saksena PN, Chaturvedi UC. Japanese encephalitis-an important cause of acute childhood encephalopathy in Lucknow, India. Postgrad Med f 1988;64:18-22.

20 Hardas UD, Pathak AA, Jahagirdar UL. Virological studies in encephalitis. Indian $\mathcal{F}$ Pediatr 1976;43:270-1. 